\section{ORIGINAL} RESEARCH

\section{F. Arrigoni}

C. Parazzini

A. Righini

C. Doneda

L.A. Ramenghi

G. Lista

F. Triulzi

\title{
Deep Medullary Vein Involvement in Neonates with Brain Damage: An MR Imaging Study
}

BACKGROUND AND PURPOSE: Different and specific MR imaging patterns of lesions involving WM are widely defined in neonatal encephalopathy. The aim of this study was to describe a novel MR imaging pattern of damage characterized by the abnormal prominence of DMVs in premature and full-term neonates.

MATERIALS AND METHODS: Twenty-one (11 premature and 10 full-term) neonates with MR imaging evidence of linear radially oriented fan-shaped lesions in the periventricular WM and without dural venous thrombosis were enrolled in this retrospective study. A total of $37 \mathrm{MR}$ imaging examinations were performed at ages ranging from day 0 to 24 months.

RESULTS: According to the appearance of linear anomalies on T2-weighted images, we identified 2 main patterns: T2 hypointense lesions without WM cavitations and T2 hypointense lesions associated with linear cysts. The first pattern was found in 17 examinations performed between 0 and 44 days of life; the second pattern was found in another 14 examinations performed between 6 days and 4 months of life. Five examinations performed between 9 and 24 months of life showed a reduction in volume and hyperintense signal intensity of the periventricular WM on T2-weighted and FLAIR images.

CoNCLUSIONS: Subtle linear WM lesions with the same anatomic distribution of DMVs may be evident in premature and full-term neonates without signs of major venous thrombosis, both in the acute and subacute phases. Their appearance and evolution suggest that transient DMV engorgement/ thrombosis may be responsible for WM damage that can lead to a PVL-like pattern.

ABBREVIATIONS: DMV = deep medullary vein; FFE = fast-field echo; GMH = germinal matrix hemorrhage; $\mathrm{PH}=$ parenchymal hematoma; $\mathrm{PVL}$ = periventricular leukomalacia; $\mathrm{US}=$ sonography

$\mathbf{N}$ eonatal cerebral damage may be different between preterm and full-term neonates because of changes in brain maturity at the time of insult.

WM is the main target of lesions in premature neonates. PVL is the most frequent pattern of injury in these patients and can be associated with GMH, intraventricular hemorrhage, and periventricular hemorrhagic infarction. ${ }^{1,2}$ On the other hand, in full-term neonates, cortical and deep gray matter is mostly affected $^{3}$; however, recent studies ${ }^{4,5}$ have also reported isolated WM lesions in $20 \%$ of term neonates with hypoxicischemic insult.

In addition, major sinovenous thrombosis with secondary involvement of WM can be observed in both premature and full-term neonates. In these cases, DMV engorgement associated with periventricular hemorrhagic infarction can often be seen on MR imaging studies. ${ }^{6}$

The increased use of early-life MR imaging has allowed us to observe a particular pattern of WM lesions suggestive of DMV involvement, without evidence of sinovenous thrombosis. The aim of this work was to report MR imaging findings and the evolution of WM injury related to DMV pathology in premature and full-term neonates.

Received February 7, 2011; accepted after revision March 24.

From the Departments of Radiology and Neuroradiology (F.A., C.P., A.R., C.D., F.T.), Children's Hospital V. Buzzi-Instituti Clinic di Perfezionamento (I.C.P.), Milan, Italy; Neonatal Intensive Care Unit (G.L.), Children's Hospital V. Buzzi-ICP, Milan, Italy; Neonatal Intensive Care Unit, (L.A.R.) Fondazion IRCCS Ca'Granda-Ospedale Maggiore Policlinico, Milan, Italy.

Please address correspondence to Filippo Arrigoni, MD, IRCCS E. Medea, Via don Luigi Monza 20, 23842, Bosisio Parini, LC, Italy; e-mail: filippo.arrigoni@bp.Inf.it http://dx.doi.org/10.3174/ajnr.A2687

\section{Materials and Methods}

\section{Patients}

The study included neonates with MR imaging evidence on T1- and T2-weighted spin-echo sequences of brain damage characterized by multiple linear WM lesions with a radial course from the surface of the ventricles toward the cortex, in the absence of major sinovenous thrombosis.

During a 4-year period (2005-2009), neonates were retrospectively selected from our MR imaging data base of 377 neonates studied in the first 2 months of life, by 4 pediatric neuroradiologists (with $2,18,14$, and 25 years of experience, respectively) blinded to the clinical history of the subjects. For each patient included in the study, full consensus was reached on the presence of linear lesions and the absence of intracranial venous sinuses thrombosis. Additional exclusion criteria were the presence of brain malformations and severe motion artifacts.

Twenty-one neonates ( 16 boys, 5 girls) were selected: Eleven patients were premature (mean gestational age at birth, $33 \pm 2.2$ weeks; range, 30-36 weeks), 10 were full-term (mean gestational age at birth, $39.3 \pm 1.5$ weeks; range, 37-41 weeks). Three patients were born from twin pregnancies.

Motivation for MR imaging assessment included the following:

- Difficult delivery with signs of fetal/neonatal distress: Apgar score $<7$ at the first minute, respiratory failure, decreased fetal movements, decreased fetal heart rate, in 12 cases ( 9 premature and 3 full-term neonates)

- Seizures within the first week of life in 8 neonates ( 1 premature and 7 full-term)

- Cerebral sonographic findings (ie, periventricular bilateral hyperechogenicity) in 1 premature neonate. 


\begin{tabular}{|c|c|c|c|c|c|c|c|c|c|}
\hline \multicolumn{10}{|c|}{ Characteristics of the patients } \\
\hline Patient & $\begin{array}{c}\mathrm{GA} \\
\text { (weeks) }\end{array}$ & MRI Indication & $\begin{array}{c}\text { MRI Timing } \\
\text { (days from birth) }\end{array}$ & $\begin{array}{c}\text { MRI } \\
\text { Findings }\end{array}$ & $\begin{array}{l}\text { Associated } \\
\text { Findings }\end{array}$ & $\begin{array}{l}\text { WM Injury } \\
\text { Location }\end{array}$ & $\begin{array}{c}\text { ADC } \\
\text { Pattern }\end{array}$ & $\begin{array}{l}\text { Sequences Used } \\
\text { for Evaluation of } \\
\text { Blood Products }\end{array}$ & $\begin{array}{c}\text { 2D MR } \\
\text { Venography }\end{array}$ \\
\hline 1 & 30 & Difficult delivery & 10 & A & GMH & $A F, F P$ & FR & T2 FFE & Yes \\
\hline 2 & 31 & Seizures & 48 & B & & AF & $\mathrm{N}$ & SWI & No \\
\hline \multirow[t]{2}{*}{3} & 31 & Difficult delivery & 9 & A & $\mathrm{GMH}, \mathrm{PH}, \mathrm{CCE}$ & $A F, F P, P T, T$ & FR & SWI & No \\
\hline & & & 58 & B & & $A F, F P, P T$ & $\mathrm{~N}$ & & \\
\hline \multirow[t]{2}{*}{4} & 31 & Difficult delivery & 4 & A & $\mathrm{GMH}$ & $A F, F P, P T$ & FR & T2 echo-planar & Yes \\
\hline & & & 80 & B & & $A F, F P, P T$ & $\mathrm{~N}$ & & \\
\hline 5 & 32 & Difficult delivery & 26 & A & $\mathrm{GMH}$ & $A F, F P, P T, T$ & $\mathrm{~N}$ & T2-FFE & Yes \\
\hline \multirow[t]{2}{*}{6} & 32 & Difficult delivery & 6 & B & $\mathrm{GMH}, \mathrm{PH}, \mathrm{TI}$ & $A F, F P$ & $\mathrm{DR}$ & T2 echo-planar & Yes \\
\hline & & & 111 & B & & $A F, F P$ & & & \\
\hline 7 & 34 & Difficult delivery & 44 & A & & $A F, F P, P T$ & $\mathrm{~N}$ & T2-FFE & Yes \\
\hline 8 & 35 & Difficult delivery & 38 & B & & $A F, F P, P T$ & $\mathrm{~N}$ & SWI & No \\
\hline 9 & 35 & Difficult delivery & 45 & B & $\mathrm{GMH}$ & $A F, F P, P T, T$ & $\mathrm{~N}$ & T2 echo-planar & No \\
\hline 10 & 36 & Pathologic US & 3 & A & GMH, CCE & $A F, F P, P T$ & FR & SWI & No \\
\hline \multirow[t]{3}{*}{11} & 36 & Difficult delivery & 0 & A & & $A F, F P, P T$ & $\mathrm{DR}$ & SWI & Yes \\
\hline & & & 18 & $A$ & & $A F, F P, P T$ & $\mathrm{~N}$ & & \\
\hline & & & 628 & PVL & & $A F, F P, P T$ & $\mathrm{~N}$ & & \\
\hline \multirow[t]{2}{*}{12} & 37 & Seizures & 14 & B & & $A F, F P, P T, T$ & $\mathrm{~N}$ & T2 echo-planar & Yes \\
\hline & & & 287 & PVL & & $A F, F P, P T, T$ & $\mathrm{~N}$ & & \\
\hline \multirow[t]{2}{*}{13} & 37 & Seizures & 6 & A & GMH, TI, CCE & $A F, F P, P T, T$ & $F R$ & SWI & Yes \\
\hline & & & 48 & B & & $A F, F P, P T, T$ & $\mathrm{~N}$ & & \\
\hline \multirow[t]{2}{*}{14} & 38 & Difficult delivery & 9 & B & & $A F, F P, P T, T$ & $F R$ & SWI, T2-FFE & Yes \\
\hline & & & 567 & PVL & & $A F, F P, P T, T$ & & & \\
\hline \multirow[t]{2}{*}{15} & 39 & Difficult delivery & 14 & $B$ & $\mathrm{PH}, \mathrm{TI}, \mathrm{CCE}$ & $A F, F P, P T, T$ & $\mathrm{DR}$ & T2 echo-planar & No \\
\hline & & & 730 & PVL & & $A F, F P, P T, T$ & & & \\
\hline \multirow[t]{2}{*}{16} & 40 & Seizures & 4 & A & & $A F, F P, P T$ & $\mathrm{FR}$ & SWI & Yes \\
\hline & & & 25 & B & & $A F, F P, P T$ & $\mathrm{~N}$ & & \\
\hline \multirow[t]{2}{*}{17} & 40 & Seizures & 3 & A & $\mathrm{PH}, \mathrm{SAH}$ & $\mathrm{AF}$ & FR & SWI & Yes \\
\hline & & & 36 & $A$ & & $\mathrm{AF}$ & & & \\
\hline \multirow[t]{2}{*}{18} & 40 & Seizures & 10 & A & & $A F, F P, P T, T$ & $\mathrm{DR}$ & T2 echo-planar & No \\
\hline & & & 26 & B & & $A F, F P, P T, T$ & $\mathrm{~N}$ & & \\
\hline \multirow[t]{2}{*}{19} & 40 & Seizures & 0 & $A$ & TI, SAH & $A F, F P, P T$ & FR & T2-FFE & Yes \\
\hline & & & 7 & A & & $A F, F P, P T$ & $\mathrm{~N}$ & & \\
\hline \multirow[t]{2}{*}{20} & 41 & Difficult delivery & 21 & B & TI, CCE & $A F, F P, P T, T$ & $\mathrm{~N}$ & SWI & No \\
\hline & & & 452 & PVL & & $A F, F P, P T, T$ & $\mathrm{~N}$ & & \\
\hline \multirow[t]{3}{*}{21} & 41 & Seizures & 0 & $A$ & & $A F, F P, P T$ & $\mathrm{~N}$ & SWI & Yes \\
\hline & & & 5 & A & & $\mathrm{AF}$ & $\mathrm{N}$ & & \\
\hline & & & 416 & Normal & & & $\mathrm{N}$ & & \\
\hline
\end{tabular}

Note:-GA indicates gestational age; MRI, MR imaging; TI, thalamic ischemia; CCE, corpus callosum edema; SAH, subarachnoid hemorrhage; $A$, pattern $A ; B$, pattern B; AF, anterior frontal $W M$; $F P$, frontoparietal WM; PT, peritrigonal WM; T, temporal WM; DR, diffusely reduced ADC values; $F R$, focally reduced (surrounding lesions) $A D C$ values; $N$, normal $A D C$ values.

Follow-up MR imaging examinations were performed in 14 patients between 5 days and 24 months after the first study. The total number of MR imaging studies was 37.

The study was approved by the institutional review board.

\section{MR Imaging Technique}

The examinations were performed on a 1.5T Achieva system (Philips Healthcare, Best, the Netherlands) with a neonatal dedicated quadrature head coil. The standard protocol for neonatal brain MR imaging in use at our institution includes axial, coronal, and sagittal T1weighted spin-echo sequences (TR/TE, 600/15 ms; section thickness, $3 \mathrm{~mm}$; FOV, $180 \mathrm{~mm}$; matrix, $256 \times 256$ ), axial and coronal T2weighted fast spin-echo sequences (TR/TE, 6000/200 ms; section thickness, $3 \mathrm{~mm}$; FOV, $180 \mathrm{~mm}$; matrix, $320 \times 320$ ), and axial diffusion-weighted sequences (TR/TE, 4000/60 ms; section thickness, $3 \mathrm{~mm}$; FOV, 210; matrix, $112 \times 256$; maximum $\mathrm{b}$ factor, $1000 \mathrm{~mm}^{2} / \mathrm{s}$ ). ADC maps were calculated off-line for each examination by using extended MR imaging software on the workstation EWS (Philips Healthcare). Regions of interest were traced on the $b=0$ images of the T2 echo-planar imaging sequence on WM lesions.
Regions of interest were automatically transferred to ADC maps to obtain ADC values.

2D MR venography (TR/TE, 19/8 ms; section thickness, $1 \mathrm{~mm}$; FOV , $180 \mathrm{~mm}$; matrix, $220 \times 180$ ) was performed in 13 patients; in the other 8 patients, possible signs of thrombosis of the main intracranial sinuses were evaluated on multiplanar conventional images.

Axial SWI (TR/TE, 35/15 ms; section thickness, $3 \mathrm{~mm}$; FOV, $180 \mathrm{~mm}$; matrix, $200 \times 160)$ was acquired in 11 patients $(14$ examinations), while axial T2-FFE images (TR/TE, 773/23 ms; section thickness, $3 \mathrm{~mm}$; FOV, $180 \mathrm{~mm}$; matrix, $256 \times 64$ ) were acquired in 5 patients. In 6 patients without both SWI and T2-FFE sequences, the presence of abnormal blood products in brain parenchyma was evaluated on the T2-weighted $b=0$ images extracted from the echoplanar diffusion-weighted sequence; all subjects underwent at least 1 of the magnetic-susceptibility-sensitive sequences (T2-FFE, SWI, or T2 echo-planar). In 4 follow-up examinations, performed at 10-15 months of age, axial and coronal FLAIR sequences (TR/TI/TE, 11 000/2800/180 ms; section thickness, 3 mm; FOV, 180 mm; matrix, $208 \times 102)$ were also obtained.

No sedation was used during scanning in patients up to 2 months 

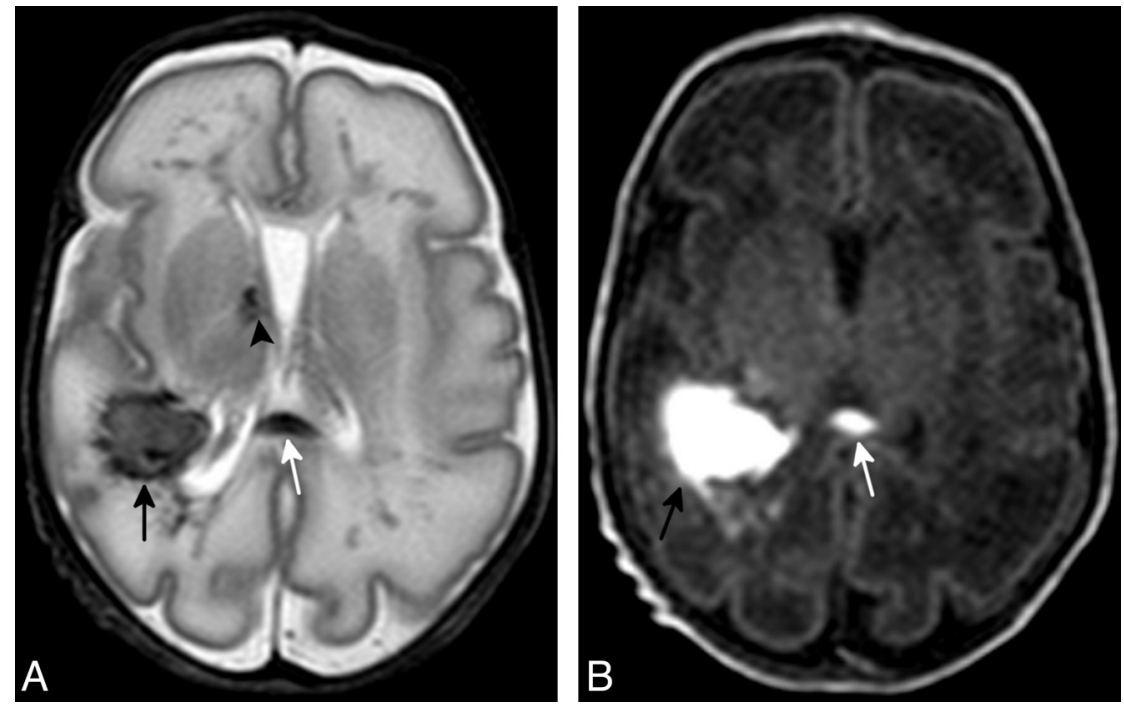

Fig 1. Axial T2-weighted $(A)$ and axial T1-weighted (B) sections in a premature neonate (gestational age, 31 weeks; case 4, Table). Periventricular WM fan-shaped linear lesions are evident bilaterally on both T2 and T1 sequences. Parietal hematoma (black arrows), GMH (arrowhead), and a lesion in the corpus callosum (white arrows) are also evident.
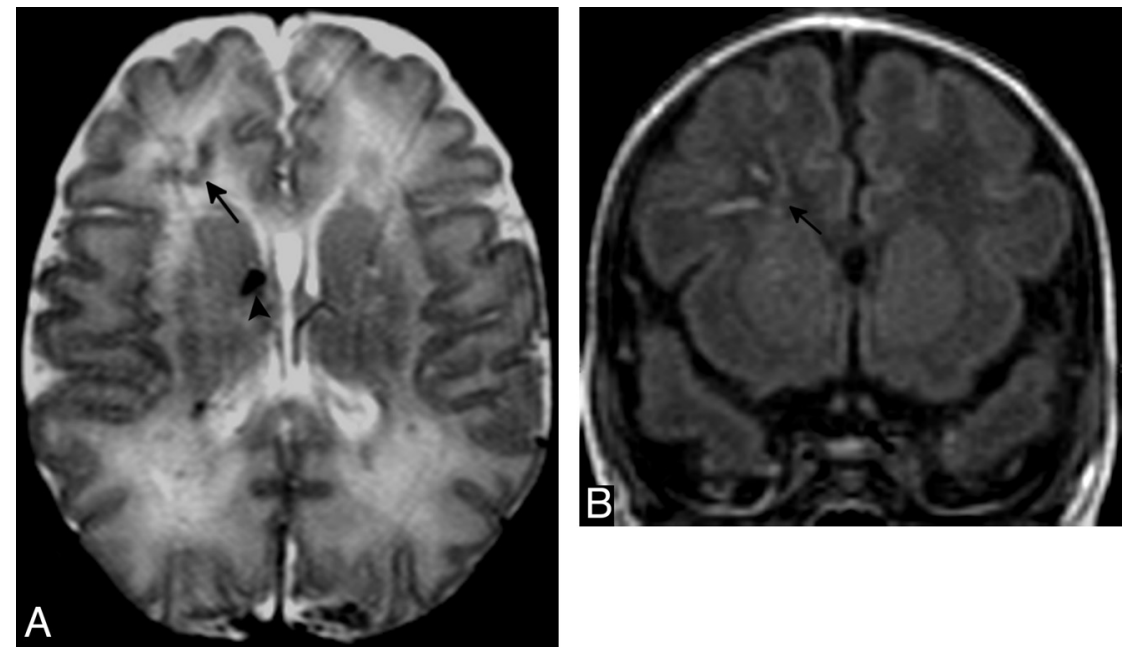

Fig 2. Axial T2-weighted $(A)$ and coronal T1-weighted (B) sections in a full-term neonate (case 18, Table). Right frontal WM linear lesions suggestive of thrombotic DMVs are shown (black arrows). Arrowhead denotes a small right GMH.

of life. Patients studied at older ages were sedated with sodium thiopental administrated through peripheral venous access; oxygen-saturation monitoring during the examination was performed through a pulse oximeter.

\section{Results}

According to the T2-weighted appearance of periventricular WM radial lesions, we identified 2 different patterns of findings (Table):

A) Linear hypointense lesions without cysts (Figs 1 and 2).

B) Linear hypointense lesions associated with linear periventricular cysts (Fig 3).

\section{Pattern A}

The first pattern was detected in 17 examinations performed in 13 patients scanned between 0 and 44 days of life (mean age at MR imaging, $10.9 \pm 12.9$ days). T1-weighted images showed linear hyperintense WM lesions corresponding to T2 linear hypointense alterations in 14 examinations, WM hyper- intense punctuate lesions in 2 examinations, and no lesions in 1 examination. The $\mathrm{ADC}$ value was reduced in the $\mathrm{WM}$ around radial lesions in 9 cases (Fig 4), diffusely reduced in the WM in 2 cases, and normal in 6 cases. Patients with abnormally reduced ADC maps were scanned between 1 and 18 days of life, while those with normal ADC maps were scanned at days $0,5,7$, and, in 3 cases, $>10$ days after birth. SWI sequences, performed in 8/17 examinations, showed small dilated periventricular radial vessels in 5 patients scanned in the first 10 days from birth (Fig 5) and normal findings in 3 patients scanned at days 4, 5, and 36 of life.

\section{Pattern B}

The second pattern was observed in 14 examinations performed in 13 patients between 6 days and 4 months of life (mean age at MR imaging, $41 \pm 29.2$ days). In 11/14 examinations, T1-weighted images also showed linear hyperintense lesions associated with linear periventricular cysts. In the other 3 cases, no signal-intensity alterations were evident on 

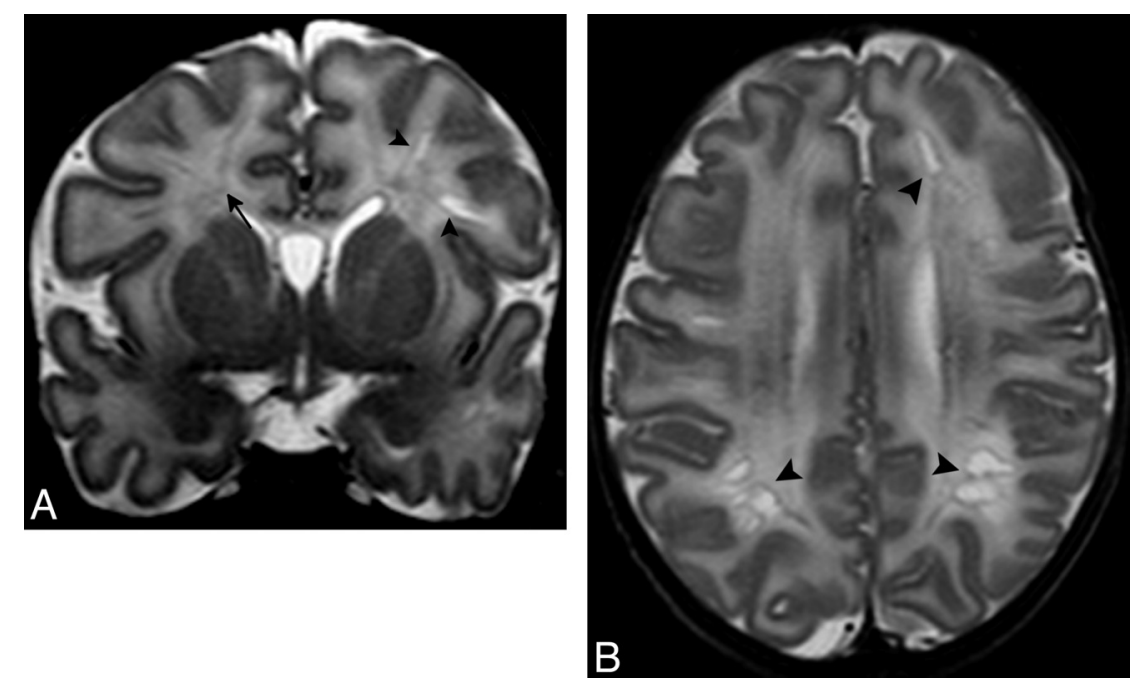

Fig 3. Coronal $(A)$ and axial $(B)$ T2-weighted sections in a full-term neonate (case 13, Table). Periventricular linear lesions (black arrows) associated with linear cysts (arrowheads) are shown.
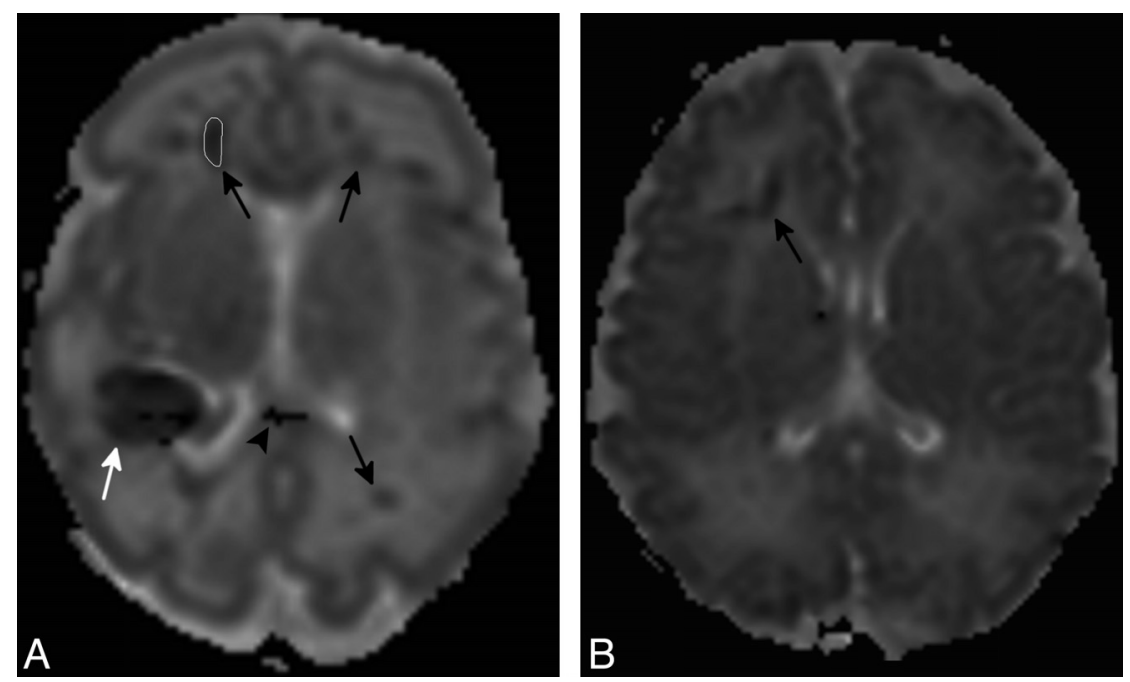

Fig 4. Axial $A D C$ maps in premature ( $A$, same case as in Fig 1$)$ and full-term ( $B$, same case as in Fig 2$)$ neonates. ADC reduction (arrows) is evident in the WM surrounding linear lesions described on T1- and T2-weighted images. A sample region of interest for ADC calculation $(A)$ has been traced in the right frontal lobe. The white arrow and arrowhead show ADC alterations corresponding to parietal hematoma and a corpus callosum lesion.

T1-weighted images. ADC values appeared reduced in the WM around the lesions in 1 patient, diffusely reduced in the WM in 2 cases, and normal in 11 cases. Abnormally low ADC values were found in patients examined in the first 15 days of life, while normal values were obtained after 20 days of life. SWI, performed in 6/14 cases, showed a dilated vessel in 1 patient at day 9 and normal findings in 5 patients studied $>20$ days from birth.

\section{Other Findings}

Lesions were bilateral in 16 patients and unilateral (right hemisphere) in 5 patients. The alterations involved periventricular WM around the frontal horns in 36 examinations (21 patients), around the ventricle body in 32 examinations (19 patients), and around the peritrigonal and periventricular occipital WM in 29 examinations (17 patients). Periventricular temporal WM was involved in 15 examinations (9 pa-

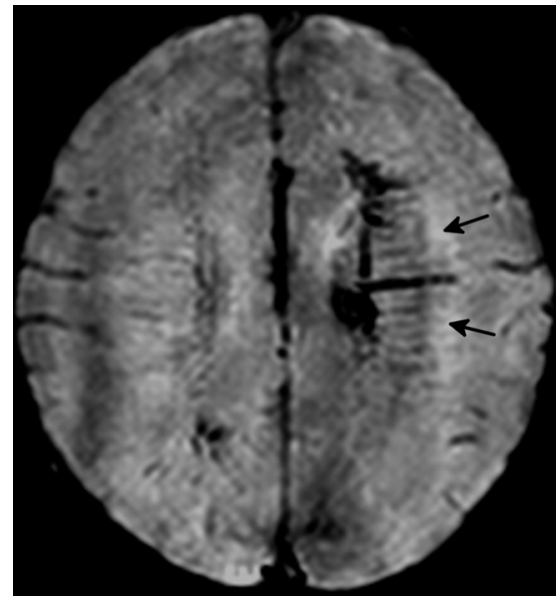

Fig 5. Axial SWI in a full-term neonate (case 14, Table) shows small dilated vessels, compatible with DMV distribution, in the left periventricular WM (black arrows). 

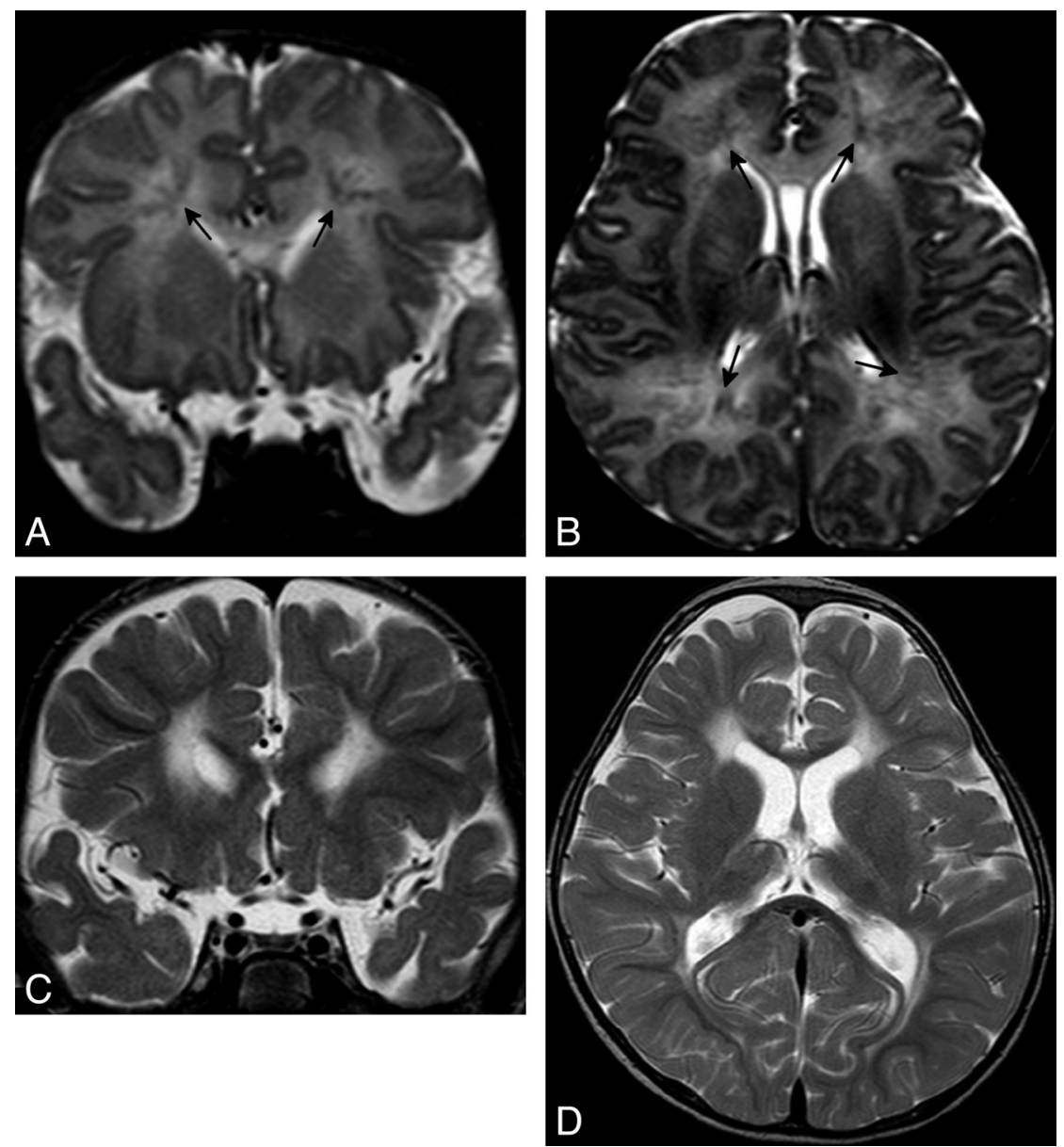

Fig 6. Coronal and axial T2-weighted sections in a full-term neonate (case 15 , Table) examined at 9 days $(A$ and $B)$ and 18 months $(C$ and $D)$ of life. Bilateral anterior and posterior periventricular radial WM lesions are due to DMV pathology in the first examination (arrows in A and B). A PVL-like pattern with hyperintense signal intensity and reduced thickness of periventricular WM is evident on follow-up $(C$ and $D)$.

tients), and the damage was usually less evident than that in other localizations in terms of signal-intensity alteration on the T2-weighted sequences.

No differences in distribution, signal intensity, and morphology of linear lesions were identified between premature and term neonates.

In 9 subjects ( 4 preterm and 5 full-term neonates), periventricular linear lesions were isolated, while in 12 subjects, MR imaging showed additional findings such as $\mathrm{GMH}$-intraventricular hemorrhage ( 1 full-term and 7 preterm neonates), PH (2 full-term and 2 preterm neonates), signs of thalamic ischemia (1 preterm and 4 full-term neonates), edematous thickening of the corpus callosum ( 3 full-term and 2 preterm neonates), and subarachnoid hemorrhage (2 full-term neonates).

MR imaging showed an evolution of the pattern of damage in $11 / 14$ patients who underwent follow-up examinations. In 5 neonates rescanned between 25 and 80 days of life, pattern $\mathrm{A}$ changed to pattern B. In 5 follow-up examinations performed between 9 and 24 months of life (mean age at MR imaging, $17.8 \pm 5.7$ months), MR imaging showed findings similar to those in PVL; in these patients, periventricular frontal, parietal, and occipital WM was reduced in thickness and hyperintense on T2-weighted and FLAIR images (Fig 6). In 1 patient, the follow-up examination performed at 14 months of age resulted in normal findings. In $3 / 14$ patients, follow-up examinations showed unchanged patterns: pattern $B$ in 1 patient at 6 and 111 days of life and pattern $A$ in 2 patients with short-term follow-up scans (1 week and 1 month).

\section{Discussion}

MR imaging evidence of WM radial linear lesions in the territory of distribution of DMVs in neonates with brain damage suggests that congestion and/or thrombosis of these vessels may be part of the pathologic chain of events in preterm and full-term neonate encephalopathy, even without associated sinus thrombosis.

This work highlights the MR imaging patterns of such phenomena in a group of 21 subjects to make neuroradiologists acquainted with this condition, which has been substantially neglected by the neuroimaging literature so far. To the best of our knowledge, this is the first MR imaging study collecting neonates with such DMV lesions in the absence of venous sinus thrombosis. However, our work cannot currently provide robust data regarding the etiologies of such lesions.

According to angiographic studies, WM venous drainage is supported by 2 main groups of veins: the superficial and deep medullary veins..$^{7-10}$ Superficial veins are small short vessels that originate $1-2 \mathrm{~cm}$ below gray matter, run in a ventricolofugal way through the cortical layer, and drain into superficial 


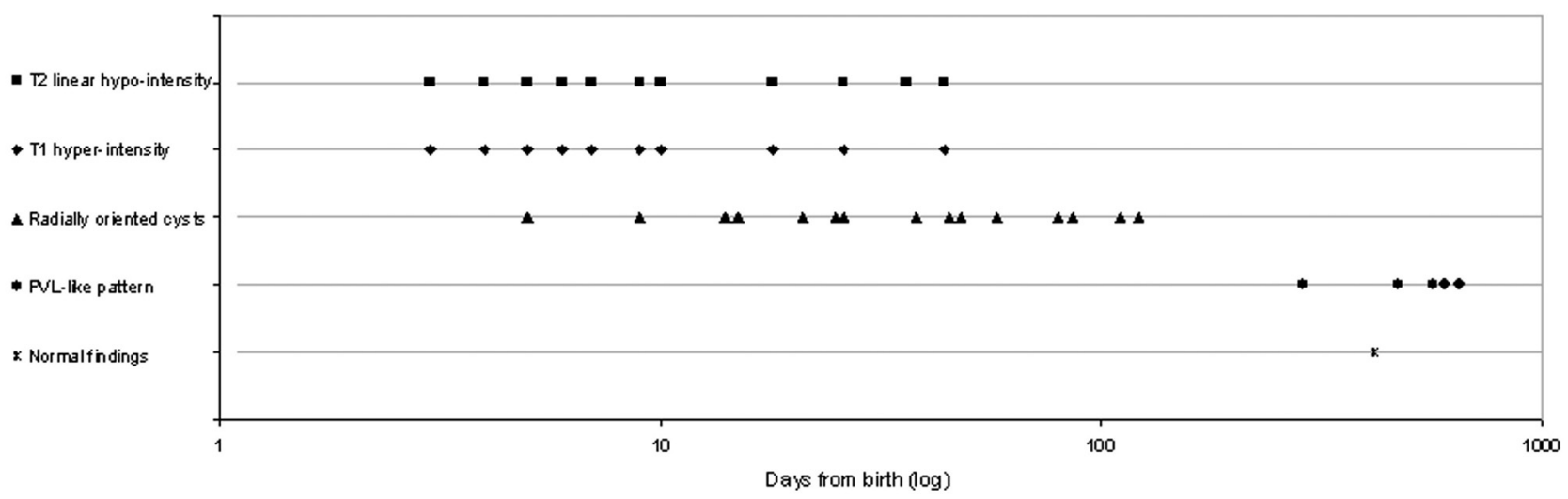

Fig 7. The graph shows the evolution of lesions with time (days in logarithmic scale).

pial veins. DMVs are longer and larger vessels that originate in the subcortical WM in the same areas as superficial veins, run in a ventricolopetal way to the ventricular surface, and drain into subependymal veins. DMVs reach the ventricle surface, converging to specific areas localized above the caudate nucleus and bifurcating posteriorly along its tail and the roof of the occipital horn. This anatomic distribution is the reason behind the fan-shaped aspect of DMVs in angiographic studies.

A third smaller group of draining veins is made up of transcerebral veins that originate from the cortical surface and run directly through WM to the subependymal veins. These vessels seem to be more common in the temporal lobes and around the occipital horns. ${ }^{11}$ In healthy subjects, both adults and neonates, conventional MR imaging cannot usually demonstrate DMVs; SWI, which is highly sensitive to deoxygenated venous blood, highlights these vessels as very subtle hypointense lines lying in the WM as described above. In adults, abnormal DMV findings have been described in pathologic conditions such as meningitis, tumors, arteriovenous malformations, developmental venous anomalies, periventricular collagenosis, extensive venous thrombosis, and Sturge-Weber syndrome. ${ }^{12,13}$ In these conditions, the observed DMVs are dilated. Some pediatric cases of SturgeWeber syndrome and chronic ischemia with DMV alterations have been reported, ${ }^{14,15}$ but little information is available about the involvement of the DMV in neonates. ${ }^{16,17}$

In this study, the patients' anatomic distributions of WM lesions along with their ventricularly converging wedgeshaped appearance make us hypothesize that these linear alterations represent pathologic DMVs. This hypothesis is supported by imaging findings on SWI performed in an acute phase. Compared with spin-echo and FFE sequences, SWI has shown the best anatomic detail and a high sensitivity (6/8 patients studied in the first 10 days of life) in detecting WM lesions as dilated vessels draining into subependymal veins. In 9 cases, the DMV lesions were isolated, in other words, without other apparent signs of brain damage on MR imaging. In 10 cases, they were associated with GMH and/or periventricular hematoma. According to these data and to other published pathology studies, ${ }^{8,18,19}$ we may assume that isolated DMV lesions, GMH, and $\mathrm{PH}$ may represent different stages of a common pathologic process related to cerebral vascular immaturity.
In premature neonates, germinal matrix vessels are highly sensitive to rupture. ${ }^{19}$ Subependymal hemorrhage and the subsequent local thrombus/hematoma could induce an increase in the upstream draining venous pressure, creating the conditions for congestion and diffuse thrombosis of small DMVs. When the insult is severe, the increased pressure may lead to rupture of the veins, and parenchymal bleeding may occur, as seen in 4 patients. A similar condition may occur during prenatal life, as reported by a recent work, ${ }^{20}$ in which central venous hypertension was considered responsible for brain damage associated with DMV involvement in fetuses with circulatory heart failure. Moreover, pathology studies ${ }^{8,18}$ confirm that DMV thrombosis may be associated with perivenular hemorrhage in premature neonates.

In full-term neonates, germinal matrix and parenchymal hemorrhages are less frequent, but DMV thrombosis may still be evident. Transient hypoperfusion or cerebral blood flow impairment may be a predisposing factor for DMV engorgement, but additional studies are needed to confirm this hypothesis.

WM damage secondary to DMV thrombosis has been explained by Nakamura et al in a postmortem study. ${ }^{11}$ The extravascular fluids accumulated in such situations might constrict arterioles and cause coagulation necrosis, directly or through chemical mediators. The ADC value reduction, observed in our patients studied in the first days after birth, indicates the presence of cytotoxic edema and, therefore, of acute cell damage. The reduction of ADC values in most of our cases (11 examinations) was limited to the areas around the linear T2 hypointensity, but in 4 cases, it involved the entire WM. We do not have sufficient data to determine if there are clinical/radiologic differences in the outcomes between these 2 classes of patients.

Lesion evolution with time is similar for all patients. In the acute phase, within the first month of life, the morphologic pattern of lesions and SWI findings suggests that DMVs are enlarged, congested, and/or thrombosed. Afterward, WM around lesions undergoes necrosis and cystic sequelae; this subacute phase lasts up to 4 months following the initial injury. Patients examined in a chronic phase (after 10 months) demonstrated that the lesions result in a volume reduction and hyperintense signal intensity of periventricular WM on T2weighted and FLAIR images, as occurs in typical PVL (Fig 7).

Unlike PVL, which primarily affects peritrigonal and deep 
parietal areas, ${ }^{21}$ in our patients, frontal/anterior WM was always involved. This observation supports the hypothesis that DMV-related WM lesions may have little in common with the pathophysiology of classic PVL, which usually occurs in preterm neonates.

Concerning lesion distribution, our study revealed that the temporal lobes were less affected than other brain regions (both in the number of patients with such lesions and in the severity of the resulting damage). Because WM in the temporal areas is normally thinner than that in other areas of the brain, the medullary veins are relatively short and may, therefore, be less prone to thrombosis than other vessels. In addition, transcerebral veins seem to be more represented in temporal lobes than in other regions, being able to supply/support medullary veins in case of engorgement.

Our study has several limitations. The main one is the lack of extensive clinical data, which prevented us from identifying common risk factors for the development of this pattern of damage. One preterm patient carried a metabolic mitochondrial disease and died at 2 years of age. Two preterm patients had systemic risk factors for thrombosis (right heart ventricle thrombosis and preterm coagulopathy), but in all the others, thrombophilic patterns were not systematically investigated. On the basis of available clinical data, only sepsis, dehydration, and major heart malformations could be excluded for all the neonates.

Furthermore, information concerning clinical therapy (ie, cooling, extracorporeal membrane oxygenation, and so forth) and long-term follow-up is lacking. Follow-up data would be particularly useful for determining whether our preterm and term neonates will present the same cognitive and motor skill impairment as those with classic PVL. Another limitation of the study is the lack of a standard MR imaging protocol for the detection of dural sinus thrombosis. However, in 18 patients, this diagnosis could be excluded on the basis of highly sensitive sequences like 2D MR angiography, SWI, and T2-FFE. ${ }^{22,23}$ In 3 patients, conventional sequences, MR imaging follow-up, and the full consensus among neuroradiologists were considered sufficient to exclude major venous thrombosis.

\section{Conclusions}

Our study demonstrates that DMV engorgement/thrombosis (even without major sinus thrombosis) may be part of the pathologic chain of events that leads to neonatal encephalopathy associated with WM lesions, both in preterm and fullterm patients. DMV thrombosis may be isolated or associated with other lesions, in particular germinal matrix and parenchymal hemorrhages. In the long-term MR imaging follow- up, DMV thrombosis could result in an atypical PVL-like pattern that also involves the frontal lobes.

Further studies on possible etiologic factors associated with DMV-related damage and on long-term outcome are definitely needed.

\section{References}

1. Volpe JJ. Brain injury in premature infants: a complex amalgam of destructive and developmental disturbances. Lancet Neurol 2009;8:110-24

2. Hill A. Hypoxic-ischemic cerebral injury in the newborn. In: Volpe JJ, ed. Neurology of the Newborn. 5th ed. Philadelphia: Saunders; 2008:279-95

3. Triulzi F, Parazzini C, Righini A. Patterns of damage in the mature neonatal brain. Pediatr Radiol 2006;36:608-20

4. Li AM, Chau V, Poskitt KJ, et al. White matter injury in term newborns with neonatal encephalopathy. Pediatr Res 2009:85-89

5. Krägeloh-Mann I, Horber V. The role of magnetic resonance imaging in elucidating the pathogenesis of cerebral palsy: a systematic review. Dev Med Child Neurol 2007;49:144-51

6. Ramenghi LA, Govaert P, Fumagalli M, et al. Neonatal cerebral sinovenous thrombosis. Semin Fetal Neonatal Med 2009;14:278-83

7. Huang YP, Wolf BS. Veins of the white matter of the cerebral hemispheres (the medullary veins). Am J Roentgenol Radium Ther Nucl Med 1964;92:739-55

8. Takashima S, Mito T, Ando Y. Pathogenesis of periventricular white matter hemorrhages in preterm infants. Brain Dev 1986;8:25-30

9. Jimenez JL, Lasjaunias $P$, Terbrugge $K$, et al. The trans-cerebral veins: normal and non-pathologic angiographic aspects. Surg Radiol Anat 1989;11:63-72

10. Kaplan HA. The transcerebral venous system. Arch Neurol 1959;1:148-52

11. Nakamura $Y$, Okudera $T$, Hashimoto $T$. Vascular architecture in white matter of neonates: its relationship to periventricular leukomalacia. J Neuropathol Exp Neurol 1994;53:582-89

12. Friedman DP. Abnormalities of the deep medullary white matter veins: MR imaging findings. AJR Am J Roentgenol 1997;168:1103-08

13. Elkeslassy A, Weill A, Miaux Y, et al. Dilatation of deep medullary veins in cortical venous occlusion due to tuberculous leptomeningitis. Neuroradiology 1997;39:705-07

14. Hu J, Yu Y, Juhasz C, et al. MR susceptibility weighted imaging (SWI) complements conventional contrast enhanced T1-weighted MRI in characterizing brain abnormalities of Sturge-Weber syndrome. J Magn Reson Imaging 2008;28:300-07

15. Takanashi J, Suzuki H, Barkovich AJ, et al. Medullary streaks: dilated medullary vessels in chronic ischemia in children. Neurology 2003;61:583-84

16. Counsell SJ, Maalouf EF, Rutherford MA. Periventricular haemorrhagic infarct in a preterm neonate. Eur J Paediatr Neurol 1999;3:25-27

17. Ramenghi LA, Gill BJ, Tanner SF, et al. Cerebral venous thrombosis, intraventricular haemorrhage and white matter lesions in a preterm newborn with factor V (Leiden) mutation. Neuropediatrics 2002;33:97-99

18. Gould SJ, Howard S, Hope PL, et al. Periventricular intraparenchymal cerebral haemorrhage in preterm infants: the role of venous infarction. $J$ Pathol 1987;151:197-202

19. Anstrom JA, Brown WR, Moody DM, et al. Subependymal veins in premature neonates: implications for hemorrhage. Pediatr Neurol 2004;30:46-53

20. Doneda C, Righini A, Parazzini C, et al. Prenatal MR imaging detection of deep medullary vein involvement in fetal brain damage. AJNR Am J Neuroradiol 2011;32:E146-9

21. Counsell SJ, Rutherford MA, Cowan FM, et al. Magnetic resonance imaging of preterm brain injury. Arch Dis Child Fetal Neonatal Ed 2003;88:F269-74

22. Leach JL, Fortuna RB, Jones BV, et al. Imaging of cerebral venous thrombosis: current techniques, spectrum of findings, and diagnostic pitfalls. Radiographics 2006:26(suppl 1):S19-43

23. Tong KA, Ashwal S, Obenaus A, et al. Susceptibility-weighted MR imaging: a review of clinical applications in children. AJNR Am J Neuroradiol 2008;29: $9-17$ 